\title{
Cluster dynamical mean-field study of strongly correlated heterostructures: Correlation-induced reduction of proximity effect
}

\author{
H. Ishida ${ }^{1}$ and A. Liebsch ${ }^{2}$ \\ ${ }^{1}$ College of Humanities and Sciences, Nihon University, Tokyo 156-8550, Japan \\ ${ }^{2}$ Institut für Festkörperforschung, Forschungszentrum Jülich, 52425 Jülich, Germany \\ (Received 22 April 2010; revised manuscript received 22 June 2010; published 12 July 2010)
}

\begin{abstract}
We present a dynamical mean-field theory (DMFT) study of strongly correlated heterostructures. In contrast to previous DMFT work on multilayered systems, which was mainly based on the single-site approximation, we investigate the role of interplanar Coulomb correlations by using cellular DMFT. Accordingly, the selfenergy matrix exhibits off-diagonal components in the layer index. As a model system we consider the single-band Hubbard model in a thin film geometry. The films can be either free standing or sandwiched between semi-infinite metallic leads. For isolated thin films, it is shown that the metal-insulator phase transition occurs either via a conventional mechanism, with a diverging imaginary part of the local self-energy, or via another one involving a discontinuous change of the real part of the off-diagonal self-energy. When the film is connected to metallic leads, the former phase transition disappears due to the normal-metal proximity effects, whereas the latter survives and significantly influences the electronic properties of the thin film. The leakage of metallic states into the Mott gap of the correlated film is greatly reduced compared to single-site DMFT calculations.
\end{abstract}

DOI: 10.1103/PhysRevB.82.045107

PACS number(s): 73.20.-r, 71.30.+h, 71.27.+a, 71.10.Fd

\section{INTRODUCTION}

Recent progress in thin-film technology such as pulsed laser deposition has enabled the synthesis of atomically flat thin films. Among them, heterostructures made by stacking ultrathin layers of perovskite crystals have been a target of intense study because of relatively small lattice mismatch among constituent bulk crystals. ${ }^{1-5}$ In particular, those including transition-metal elements with a partially filled $d$ shell have attracted much attention because of interesting phenomena induced by strong Coulomb interactions and by additional physical effects peculiar to layered systems, such as electron confinement in thin layers, band filling change due to charge transfer across the interface, and structural relaxations near boundary layers. ${ }^{6-10} \mathrm{~A}$ well-known example is the formation of a conducting layer at the interface between a Mott insulator $\mathrm{LaTiO}_{3}$ and a band insulator $\mathrm{SrTiO}_{3}$ as reported in the pioneering work of Ohtomo et al. ${ }^{6}$

Theoretical research on strongly correlated electrons in inhomogeneous layered systems was initiated by Potthoff and Nolting, ${ }^{11,12}$ who studied the metal-insulator transition (MIT) at the surface of the single-band Hubbard model by using the single-site dynamical mean-field theory (DMFT). ${ }^{13,14}$ Within this approximation, the electron selfenergy for each layer is determined by solving a separate many-body impurity problem. Nevertheless, since the Weiss mean-field for each layer, which is derived from the lattice Green's function of the whole system, depends on the selfenergies of the neighboring layers, the self-consistent equations for determining the self-energies of all layers are coupled. Stimulated by recent experiments on strongly correlated heterostructures, theoretical studies of inhomogeneous layered systems were performed for a number of systems by employing tight-binding model Hamiltonians with a Hubbard-like Coulomb repulsion term, and in some cases, also with the long-range Hartree potential term accounting for charge redistributions across the layers. ${ }^{15-30}$ The main focus of these DMFT studies were heterostructures for a thin film sandwiched between two bulk systems ${ }^{16-21}$ and interfaces between two bulk systems. ${ }^{22-24}$ Most of these studies adopt single-site DMFT. An exception is the cellular DMFT $(\text { CDMFT })^{31}$ study of Yunoki et al. ${ }^{24}$ who used a four-site cluster within each layer in solving the quantum impurity problem and explored the possibility of magnetic ordering and superconductivity at the interface between undoped high- $T_{c}$ cuprates and manganites. Spatial fluctuations between layers were neglected. The role of interlayer correlations was, however, studied within a bilayer t-J model by Heindl et $\mathrm{al}^{25}$

In the present work, we pursue another direction by studying the effect of interlayer electron correlations in inhomogeneous layered systems with strong Coulomb interactions. In principle, of course, intersite correlations both parallel and perpendicular to the planes comprising a heterostructure might be important. In view of the exponentially growing numerical effort, however, it is presently necessary to focus on relatively small clusters consisting of only few sites. Correlations in the normal direction to the layer structure are of particular interest because of the proximity effect at the interface between strongly and weakly correlated materials. For instance, metallic states at the Fermi level of one system can spill over into the gap of an adjacent Mott insulator. Also, the correlation induced effective mass enhancement in one compound can give rise to mass enhancement in a neighboring weakly correlated metal. Since these phenomena have been studied so far only within single-site DMFT, it is of great interest to investigate to what extent these kinds of proximity effects are modified by spatial fluctuations in the normal direction. Intersite correlations within planes parallel to the interface are neglected in the present work.

As in previous works, we employ a single-band Hubbard Hamiltonian to model the system, but perform CDMFT cal- 
culations, where a one-dimensional (1D) chain oriented normal to the layer plane is chosen as a cluster in solving the quantum impurity problem. Thus, while interatomic electron correlations within the same layer are ignored, the offdiagonal elements of the electron self-energy between neighboring layers are fully taken into consideration. As a model system, we consider a sandwich structure in which a thin layer of a strongly correlated material is connected to noninteracting metallic leads on both sides. As a reference system, we also study the electronic structure of free-standing films not attached to metallic leads. The focus here is on spatial fluctuations within the strongly correlated material of the heterostructure. Fluctuations across the interface between strongly and weakly correlated systems are also interesting, but will not be addressed in this paper.

The main result of this work is that the presence of interlayer electron correlations qualitatively changes the nature of the MIT in a strongly correlated thin film. For isolated films not attached to metallic leads, an insulating gap can be opened via a mechanism characterized by a discontinuous change in the real part of the off-diagonal self-energy between nearest-neighbor layers. This is in contrast to a more conventional MIT where the divergence of the imaginary part of the local self-energy induces the insulating gap. Furthermore, while the MIT via the conventional mechanism disappears due to the normal-metal proximity effects when the film is connected to metallic leads, ${ }^{23}$ the phase transition with a discontinuous change in the real part of the selfenergy continues to exist even in the presence of metallic leads, giving rise to electronic properties which are qualitatively different from those obtained from a single-site DMFT treatment. In particular, we demonstrate that the penetration of metallic lead states into the Mott gap of the correlated film is strongly reduced compared to the one derived within single-site DMFT. Moreover, as a consequence of interplanar correlations, the critical Coulomb energies are considerably lower than those obtained within local DMFT.

These results indicate that spatial fluctuations between the planes of a heterostructure are of similar significance as intraplanar fluctuations in two-dimensional (2D) correlated systems. ${ }^{32-34}$ Results derived within inhomogeneous singlesite DMFT may therefore serve for qualitative purposes, but should be revised considerably, both with respect to the nature of the Coulomb driven metal-insulator transition and the magnitude of critical Coulomb energies.

The outline of this paper is as follows. In Sec. II, we describe the present model and discuss several theoretical details concerning our CDMFT calculations. In Sec. III, we present the numerical results and analyze them with particular focus on the difference obtained within CDMFT and single-site DMFT treatments. Section IV contains the summary.

\section{THEORY}

We consider inhomogeneous layered systems made by stacking the (001) planes of a simple cubic lattice with lattice constant $a$ in the $z$ direction. The $j$ th layer is located at $z_{j}$ $=j a$, and the $2 \mathrm{D}$ lattice vector in the plane is denoted by $\mathbf{x}$ whose $x$ and $y$ components are multiples of $a$. We use index $p=\left(\mathbf{x}, z_{j}\right)$ to specify an atomic site in the system. For simplicity, we consider the single-band Hubbard model. The single-electron part of the Hamiltonian is

$$
\hat{h}=\sum_{p \sigma} \epsilon_{p} \hat{n}_{p \sigma}-\sum_{\left\langle p p^{\prime}\right\rangle \sigma}\left(t_{p p^{\prime}} c_{p \sigma}^{\dagger} c_{p^{\prime} \sigma}+\text { H.c. }\right),
$$

while the interaction part is given by

$$
\hat{v}=\sum_{p} U_{p} \hat{n}_{p \uparrow} \hat{n}_{p \downarrow},
$$

where $c_{p \sigma}\left(c_{p \sigma}^{\dagger}\right)$ is the electron annihilation (creation) operator at site $p$ with spin $\sigma, \hat{n}_{p \sigma}=c_{p \sigma}^{\dagger} c_{p \sigma}$, and the summation in the second term in Eq. (1) is taken over pairs of nearestneighbor sites. Throughout the present paper, we will denote the operator corresponding to a physical quantity $A$ by $\hat{A}$ and its matrix elements by $A_{p p^{\prime}}$. All sites within a layer are assumed to be equivalent. We label the site energy of layer $j$ as $\boldsymbol{\epsilon}_{j}$, the Coulomb energy of layer $j$ as $U_{j}$, the $x$ and $y$ components of the in-plane transfer integrals in layer $j$ as $t_{j}^{x}$ and $t_{j}^{y}$, and the transfer integral between two nearest-neighbor layers, $j$ and $j^{\prime}=j \pm 1$, as $t_{j j^{\prime}}^{z}$

We like to calculate the finite-temperature Green's function of the full Hamiltonian $\hat{\mathcal{H}}=\hat{h}+\hat{v}$,

$$
G_{p \sigma, p^{\prime} \sigma^{\prime}}\left(i \omega_{n}\right)=\left\langle p \sigma\left|\left[i \omega_{n}+\mu-\hat{h}-\hat{\Sigma}\left(i \omega_{n}\right)\right]^{-1}\right| p^{\prime} \sigma^{\prime}\right\rangle,
$$

where $\mu$ denotes the chemical potential of the system, $\omega_{n}$ $=(2 n+1) \pi k_{B} T$ are Matsubara frequencies at temperature $T$, and $\hat{\Sigma}\left(i \omega_{n}\right)$ denotes the frequency-dependent self-energy describing the effect of Coulomb interactions. In the present work we consider only paramagnetic systems and omit hereafter spin indices for simplicity.

Furthermore, we exploit translational symmetry in the planes. By introducing the 2-D wave vector $\mathbf{k}(-\pi / a$ $\left.\leq k_{x}, k_{y} \leq \pi / a\right)$, the lattice Green's function Eq. (3) can be expressed as

$$
\begin{aligned}
G_{p p^{\prime}}\left(i \omega_{n}\right)= & \int \frac{d \mathbf{k}}{(2 \pi)^{2}} e^{i \mathbf{k} \cdot\left(\mathbf{x}-\mathbf{x}^{\prime}\right)} \\
& \times\left\langle j\left|\left[i \omega_{n}+\mu-\hat{H}\left(\mathbf{k}, i \omega_{n}\right)\right]^{-1}\right| j^{\prime}\right\rangle,
\end{aligned}
$$

where $p=\left(\mathbf{x}, z_{j}\right), p^{\prime}=\left(\mathbf{x}^{\prime}, z_{j^{\prime}}\right)$, and the Hamiltonian matrix elements in the mixed representation are given by

$$
H_{j j^{\prime}}\left(\mathbf{k}, i \omega_{n}\right)=E_{j}(\mathbf{k}) \delta_{j j^{\prime}}-t_{j j^{\prime}}^{z}+\Sigma_{j j^{\prime}}\left(\mathbf{k}, i \omega_{n}\right),
$$

where

$$
E_{j}(\mathbf{k})=\epsilon_{j}-2 t_{j}^{x} \cos \left(k_{x} a\right)-2 t_{j}^{y} \cos \left(k_{y} a\right) .
$$

We consider isolated thin films consisting of $N$ atomic layers as well as those attached to semi-infinite bulk systems (see Fig. 1). For the latter, we treat explicitly only the central region made out of $N$ layers $(1 \leq j \leq N)$. The effects of the semi-infinite left lead $(j<1)$ and right lead $(j>N)$ on the sides of the central system are expressed by adding tightbinding embedding potential terms $\mathrm{s}^{30,35}$ to Eq. (5). In principle, the embedded region could also include the first or first 


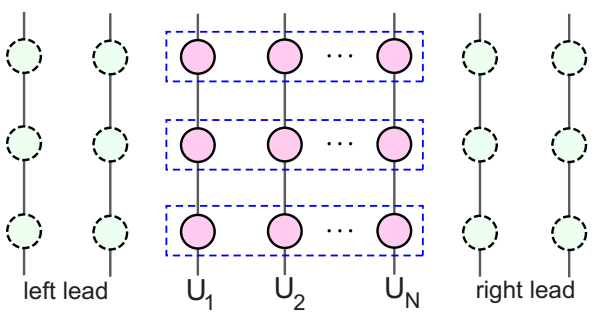

FIG. 1. (Color online) Tight-binding model for inhomogeneous layered systems. The central system made out of $N$ layers is treated explicitly, while the effects of the left and right leads are incorporated by using the embedding potentials acting on the boundary layers. Interplanar Coulomb correlations are included within 1D atomic chains indicated by dashed rectangles. These chains form the $N$-site clusters used in the CDMFT calculation. The heterostructure is translationally invariant in the parallel direction.

few planes of the leads, making their self-energies also layer dependent. We neglect this effect here and assume all layers of the semi-infinite leads to be noninteracting. Thus,

$$
\begin{aligned}
H_{j j^{\prime}}\left(\mathbf{k}, i \omega_{n}\right)= & E_{j}(\mathbf{k}) \delta_{j j^{\prime}}-t_{j j^{\prime}}^{z}+\Sigma_{j j^{\prime}}\left(\mathbf{k}, i \omega_{n}\right)+s_{j j^{\prime}}^{L}\left(\mathbf{k}, i \omega_{n}\right) \\
& +s_{j j^{\prime}}^{R}\left(\mathbf{k}, i \omega_{n}\right),
\end{aligned}
$$

where $1 \leq j, j^{\prime} \leq N$, and $s_{j j^{\prime}}^{L}\left(s_{j j^{\prime}}^{R}\right)$ are the matrix elements of the embedding potential representing the left (right) lead. We employ the nearest-neighbor tight-binding Hamiltonian in the same form as Eq. (1) to represent semi-infinite noninteracting leads. In this case the only nonvanishing matrix element for the left lead is

$$
s_{11}^{L}(\mathbf{k}, \epsilon)=\left(t_{01}^{z}\right)^{2} g_{00}^{L}\left[\epsilon-E_{L}(\mathbf{k})\right],
$$

where

$$
E_{L}(\mathbf{k})=\epsilon_{L}-2 t_{L}^{x} \cos \left(k_{x} a\right)-2 t_{L}^{y} \cos \left(k_{y} a\right),
$$

with $\epsilon_{L}, t_{L}^{x}$, and $t_{L}^{y}$ being the site-energy and parallel components of transfer integrals in the left lead. In Eq. (8), $g_{j j^{\prime}}^{L}$ $\left(j, j^{\prime} \leq 0\right)$ denotes the Green function of a semi-infinite tightbinding chain with nearest-neighbor transfer integral, $-t_{L}^{z}$, whose surface element is given by ${ }^{36}$

$$
g_{00}^{L}(w)=\frac{w-\sqrt{w^{2}-4\left(t_{L}^{z}\right)^{2}}}{2\left(t_{L}^{z}\right)^{2}} .
$$

Similarly, the matrix elements of the right-lead embedding potential vanish except for $s_{N N}^{R}(\mathbf{k}, \epsilon)$, which has the same form as Eq. (8) with the tight-binding parameters replaced by those corresponding to the right lead.

To determine the self-energy matrix, we apply CDMFT, ${ }^{31}$ i.e., the system is divided into a periodic array of clusters consisting of several atomic sites. The off-diagonal components of the self-energy within each cluster are fully taken into consideration, while those between two sites on neighboring clusters are assumed to vanish. As shown in Fig. 1, we choose the 1D atomic chain consisting of $N$ atomic sites oriented in the $z$ direction as a unit cluster. In this case the self-energy matrix elements in Eq. (3), $\Sigma_{p p^{\prime}}\left(i \omega_{n}\right)$, become nonvanishing only for those with $\mathbf{x}=\mathbf{x}^{\prime}$, resulting in a k-independent self-energy matrix in the mixed representation. On the other hand, in contrast to previous DMFT studies for multilayered systems, we fully incorporate the offdiagonal components of the self-energy between different layers. Thus, by removing index $\mathbf{k}$, the self-energy matrix elements in Eqs. (5) and (7) are written as $\Sigma_{j j^{\prime}}\left(i \omega_{n}\right)$.

By setting $\mathbf{x}=\mathbf{x}^{\prime}=0$ in Eq. (4), we obtain the interacting lattice Green's function of the cluster located at $\mathbf{x}=0$,

$$
G_{j j^{\prime}}\left(i \omega_{n}\right)=\int \frac{d \mathbf{k}}{(2 \pi)^{2}}\left\langle j\left|\left[i \omega_{n}+\mu-\hat{H}\left(\mathbf{k}, i \omega_{n}\right)\right]^{-1}\right| j^{\prime}\right\rangle .
$$

The bath Green's function determining the Weiss mean-field Hamiltonian is obtained by removing the self-energy matrix from this lattice Green's function as,

$$
G_{j j^{\prime}}^{0}\left(i \omega_{n}\right)=\left[\hat{G}^{-1}\left(i \omega_{n}\right)+\hat{\Sigma}\left(i \omega_{n}\right)\right]_{j j^{\prime}}^{-1},
$$

where all the quantities on both sides are $N \times N$ matrices defined for atomic sites in the cluster at $\mathbf{x}=0$. The next task is to solve the many-body impurity problem in which the local Coulomb interactions of the form Eq. (2) are added to the $N$-site cluster at $\mathbf{x}=0$ whereas the rest of the system is represented by a non-interacting Hamiltonian corresponding to the Weiss mean-field.

As impurity solver, we adopt the finite-temperature exact diagonalization (ED) technique ${ }^{37}$ in which $\hat{G}^{0}$ in Eq. (12) is approximated by the Green's function of an isolated cluster consisting of $N$ impurity levels plus $n_{b}$ bath levels as,

$$
G_{j j^{\prime}}^{0}\left(i \omega_{n}\right) \approx G_{j j^{\prime}}^{c l, 0}\left(i \omega_{n}\right)=\left[i \omega_{n}+\mu-\hat{h}^{c l}\left(i \omega_{n}\right)\right]_{j j^{\prime}}^{-1},
$$

where

$$
h_{j j^{\prime}}^{c l}\left(i \omega_{n}\right)=\widetilde{E}_{j} \delta_{j j^{\prime}}-\widetilde{t}_{j j^{\prime}}^{z}+\sum_{k=1}^{n_{b}} \frac{V_{j k} V_{k j^{\prime}}}{i \omega_{n}-\widetilde{\epsilon}_{k}} .
$$

Here $\widetilde{E}_{j}(1 \leq j \leq N)$ represent impurity levels, $\tilde{t}_{j j^{\prime}}$ are transfer integrals among $N$ impurity levels, $\widetilde{\epsilon}_{k}$ are bath levels, and $V_{j k}$ specify the hybridization matrix between the impurity and bath levels. These fitting parameters are chosen such that $\hat{G}^{c l, 0}$ approximates $\hat{G}^{0}$ as accurately as possible at the Matsubara frequencies on the imaginary energy axis. We then calculate $\hat{G}^{c l}$, the interacting Green's function of the cluster, in which the onsite Coulomb interaction term of the form Eq. (2) is added to the non-interacting Hamiltonian $\hat{h}^{c l}$. Numerical details concerning the calculation of $\hat{G}^{c l}$ are described in Ref. 38. Finally, we determine the self-energy matrix of the $N$-site impurity via

$$
\Sigma_{j j^{\prime}}^{c l}\left(i \omega_{n}\right)=\left(G^{c l, 0}\right)_{j j^{\prime}}^{-1}-\left(G^{c l}\right)_{j j^{\prime}}^{-1} .
$$

The key assumption in DMFT is now that this impurity selfenergy is a physically reasonable representation of the lattice self-energy. Thus, $\hat{\Sigma}^{c l}\left(i \omega_{n}\right)$ in Eq. (15) is used as the input self-energy in Eq. (11) for the next iteration. This procedure is iterated until the difference between the input and output self-energy matrices becomes sufficiently small.

For comparison, we also perform a single-site inhomogeneous DMFT calculation where the self-energy matrix in 
Eqs. (5) and (7) is assumed to be layer diagonal, i.e., $\Sigma_{j j^{\prime}}\left(i \omega_{n}\right)=\Sigma_{j j}\left(i \omega_{n}\right) \delta_{j j^{\prime}}$. For each layer $j(1 \leq j \leq N)$, one calculates the lattice Green function,

$$
G_{j j}\left(i \omega_{n}\right)=\int \frac{d \mathbf{k}}{(2 \pi)^{2}}\left\langle j\left|\left[i \omega_{n}+\mu-\hat{H}\left(\mathbf{k}, i \omega_{n}\right)\right]^{-1}\right| j\right\rangle,
$$

and further, the bath Green's function defined by

$$
G_{j j}^{0}\left(i \omega_{n}\right)=\left[G_{j j}^{-1}\left(i \omega_{n}\right)+\Sigma_{j j}\left(i \omega_{n}\right)\right]^{-1} .
$$

As in the case of CDMFT, we employ the ED technique ${ }^{37}$ and approximate $G_{j j}^{0}$ by the non-interacting Green function of an isolated cluster consisting of a single impurity level and $n_{b}$ bath levels as,

$$
G_{j j}^{0}\left(i \omega_{n}\right) \approx G_{j j}^{c l, 0}\left(i \omega_{n}\right)=\left[i \omega_{n}+\mu-h_{j j}^{c l}\left(i \omega_{n}\right)\right]^{-1},
$$

where

$$
h_{j j}^{c l}\left(i \omega_{n}\right)=\widetilde{E}_{j}+\sum_{k=1}^{n_{b}} \frac{V_{j k} V_{k j}}{i \omega_{n}-\widetilde{\epsilon}_{k}} .
$$

Then, by adding the Coulomb interaction with strength $U_{j}$ on the impurity site, one calculates the interacting Green's function of the cluster, $G_{j j}^{c l}$, for all layers. Thus, instead of solving the $N$-site impurity problem, one solves $N$ independent single-impurity problems. The output impurity self-energy of layer $j$ is evaluated as

$$
\Sigma_{j j}^{c l}\left(i \omega_{n}\right)=\left(G_{j j}^{c l, 0}\right)^{-1}-\left(G_{j j}^{c l}\right)^{-1},
$$

which is used in calculating the next lattice Green's function via Eq. (16). The iteration procedure is repeated until input and output self-energies converge to a required accuracy.

\section{RESULTS AND DISCUSSION}

The model outlined above may, in principle, be used to study charge transfer at the interface by adjusting the singleelectron Hamiltonian parameters. To focus on interlayer correlation effects, however, we consider here the special case in which all transfer integrals have the same value, i.e., $t_{j}^{x, y}$ $=t_{j j^{\prime}}^{z}=t$, including the central layers and both leads. Thus, without Coulomb interactions, the system represents a bulk simple cubic lattice characterized by a transfer integral $t$, which will be chosen as the unit of energy. For simplicity, we further choose the site energy $\epsilon_{j}$ as $-U_{j} / 2$ and the chemical potential $\mu$ as 0 , so that the system is half-filled and particlehole symmetric. In the strongly correlated layers $(1 \leq j \leq N)$, we assume $U_{j}=U$. The system therefore is characterized by three parameters: $U, N$, and $\beta=\left(k_{B} T\right)^{-1}$. In the following we present results for a low temperature, $\beta=100$.

In applying the ED formalism we use $n_{b}=3 N$ bath levels to represent the bath Green's function. Thus, the total number of orbitals in the ED calculation amounts to $N+n_{b}=4 N$. With our present numerical capability, the maximum number of layers that can be treated by the full CDMFT scheme is $N=3$ (12 orbitals). Nevertheless, our CDMFT results for $N$ $=2$ and 3 demonstrate that spatial fluctuations between layers give rise to a much richer physics than the scenario obtained within single-site DMFT. For the single-site DMFT, we use
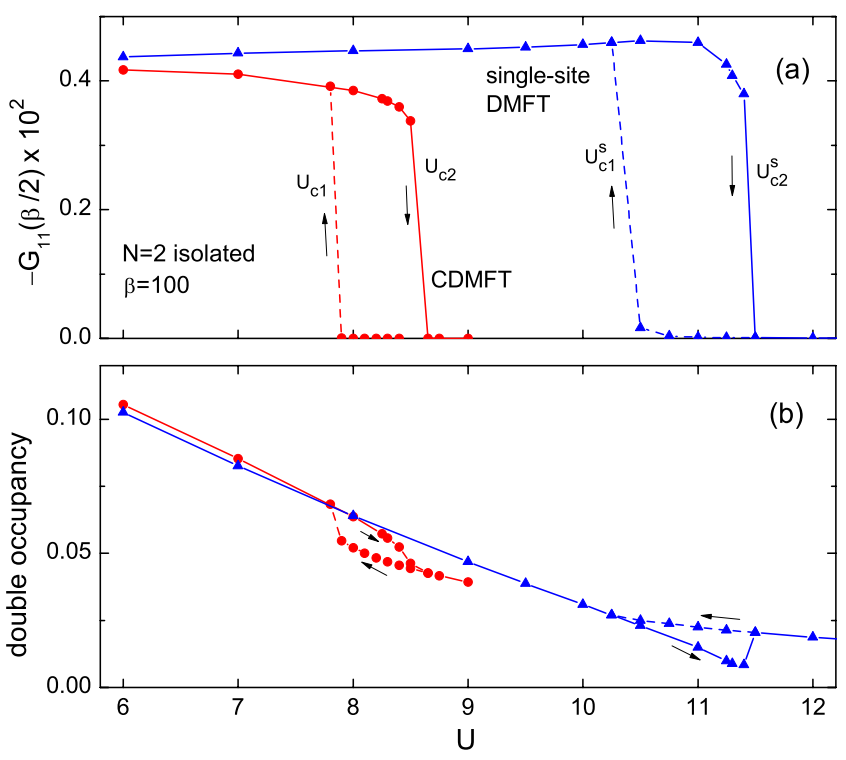

FIG. 2. (Color online) (a) $G_{11}(\beta / 2)$, imaginary-time Green's function at $\tau=\beta / 2$ and (b) double occupancy of an atomic site in the first layer $(j=1)$ for an isolated two-layer film as a function of Coulomb energy $U$ at $\beta=100$. Solid circles and triangles correspond to cluster and single-site DMFT calculations, respectively. Note the hysteresis behavior for increasing and decreasing $U$ as indicated by the arrows.

$n_{b}=3$ bath levels for each layer. While this choice slightly favors the insulating solution over the metallic one and does not yet achieve fully converged critical Coulomb energies for the MIT, it is the most suitable one for the purpose of comparing single-site and cluster DMFT results.

\section{A. Isolated $N=2$ film}

We begin with a two-layer film not attached to metallic leads. The effects of Coulomb interactions are expressed in terms of the electron self-energy. For the present half-filled two-layer film with particle-hole symmetry, $\mathfrak{R} \Sigma_{11}=\mathfrak{R} \Sigma_{22}$ $=U / 2, \mathfrak{I} \Sigma_{12}=0$, while $\mathfrak{I} \Sigma_{11}=\mathfrak{I} \Sigma_{22}$ and $\mathfrak{R} \Sigma_{12}$ are nontrivial functions of Matsubara frequency. Also, the self-energy is symmetric with respect to layer indices, i.e., $\Sigma_{i j}=\Sigma_{j i}$.

To discuss the MIT induced by onsite Coulomb interactions, we plot in Fig. 2(a) the imaginary-time Green's function at $\tau=\beta / 2$, which is related to the quasiparticle density of states (DOS), i.e., the imaginary part of the retarded Green's function via

$$
G_{j j}(\beta / 2)=\pi^{-1} \int d \epsilon F(\epsilon) \Im G_{j j}(\epsilon) .
$$

Here, $F(\epsilon)=0.5 / \cosh [\beta(\epsilon-\mu) / 2]$ is a distribution of width $4 \ln (2+\sqrt{3}) / \beta$ centered about the chemical potential $\mu$. $-G_{j j}(\beta / 2)$ represents the integrated spectral weight within a few $k_{B} T$ of $\mu$ and drops from a finite value to zero when the system undergoes a MIT. As the second criterion we plot in Fig. 2(b) the double occupancy of an atomic site, $\left\langle\hat{n}_{p \uparrow} \hat{n}_{p \downarrow}\right\rangle$. Within single-site DMFT, the MIT occurs at $U_{c 2}^{s}$ for increasing $U$ and at $U_{c 1}^{s}$ for decreasing $U$. This hysteresis behavior 

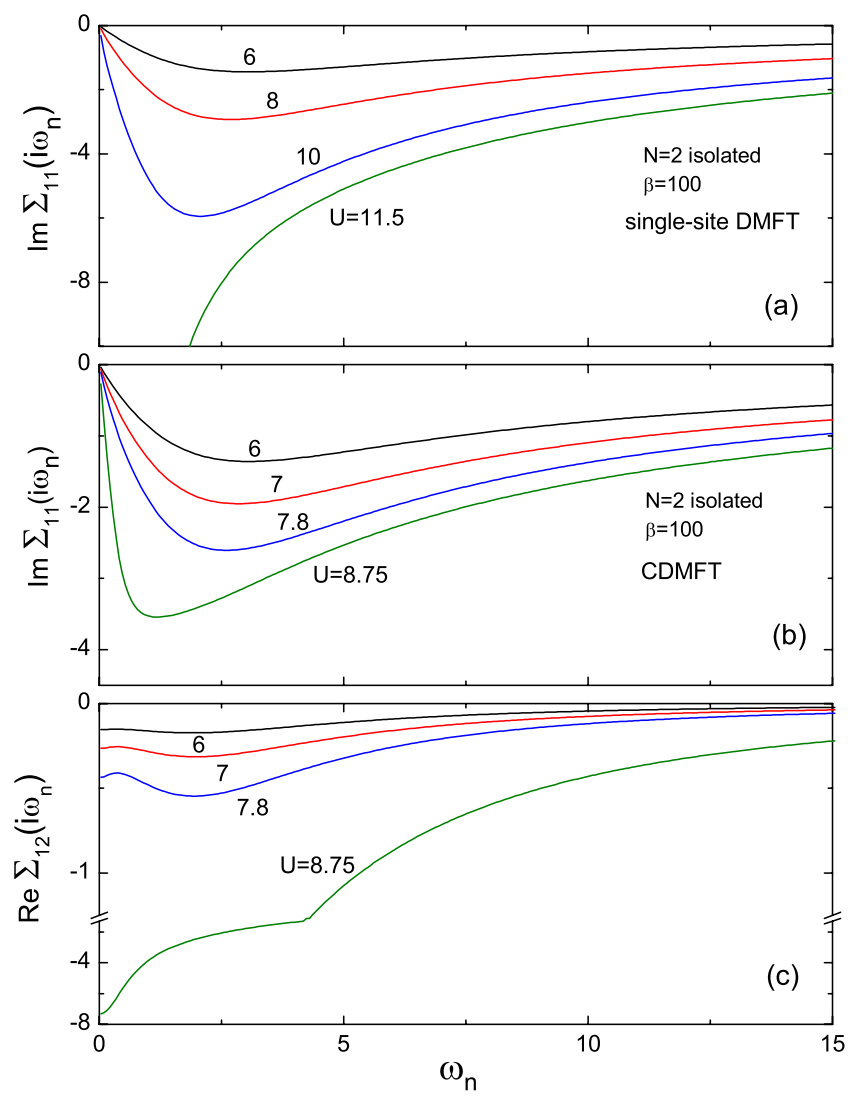

FIG. 3. (Color online) Self-energy components of an isolated 2-layer film as a function of Matsubara frequency $\omega_{n}$ at $\beta=100$. (a) $\Im \Sigma_{11}$ within single-site DMFT. (b) $\mathfrak{I} \Sigma_{11}$ and (c) $\mathfrak{R} \Sigma_{12}$ within CDMFT. Note the break in the vertical scale at -1.3 in (c).

is characteristic of a first-order phase transition. Within CDMFT, the MIT occurs at considerably lower Coulomb energies, $U_{c 1}$ and $U_{c 2}$. This trend is consistent with a recent CDMFT study of the 2D Hubbard model, where the critical $U$ for the gap opening was found to decrease with increasing cluster size. ${ }^{33}$

Even more significant than this overall lowering of critical Coulomb energies is the fact that the nature of the MIT within single-site and cluster DMFT differs qualitatively. To illustrate this point, we first show in Fig. 3(a) the imaginary part of $\Sigma_{11}$ as a function of Matsubara frequency obtained within single-site DMFT. It exhibits a characteristic behavior similar to the one found in single-site DMFT studies of bulk systems. In the metallic phase, $\Im \Sigma_{11}$ is proportional to $\omega_{n}$ at small $\omega_{n}$, with its slope in the limit of $\omega_{n} \rightarrow 0$ determining the quasiparticle weight $Z$. On the other hand, when $U$ crosses the critical value, $\Im \Sigma_{11}$ diverges as $1 / \omega_{n}$, resulting in the opening of an insulating gap at $\mu$. In contrast to these singlesite DMFT results, CDMFT yields a rather different picture. As shown in Fig. 3(b), $\Im \Sigma_{11}$ does not diverge but remains proportional to $\omega_{n}$ at small $\omega_{n}$, even when $U$ is in the insulating range $(U=8.75)$. Instead, as can be seen in Fig. 3(c), the opening of the gap in CDMFT is induced by an abrupt change in the real part of the interlayer component of the self-energy, $\mathfrak{R} \Sigma_{12}$. To see this more clearly, we show in Fig. 4(a) $\Re \Sigma_{12}\left(i \omega_{0}\right)$ as a function of $U$, where $\omega_{0}=\pi k_{B} T$ denotes the first Matsubara frequency. Evidently, this quantity exhib-
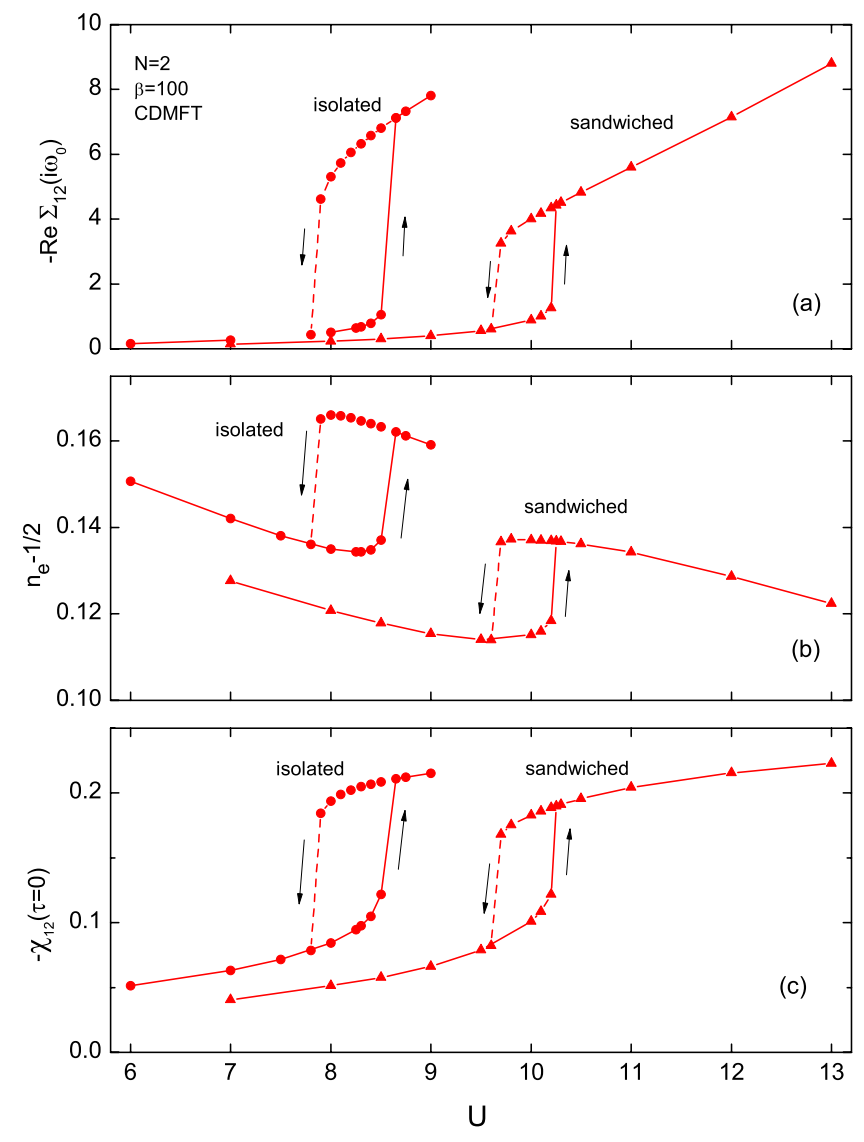

FIG. 4. (Color online) (a) $\mathfrak{R} \Sigma_{12}$ at the first Matsubara frequency $\omega_{0}=\pi / \beta$ (b) $n_{e}$, occupation of the molecular orbital $|e\rangle$, minus $1 / 2$, and (c) $\chi_{12}(\tau=0)$, interlayer spin correlation at $\tau=0$, as functions of $U$ at $\beta=100$ within CDMFT. Solid circles and triangles correspond to the isolated and sandwiched two-layer films, respectively.

its a discontinuous change at the critical Coulomb energies.

For $N=2$, it is useful to introduce the molecular orbital (MO) basis,

$$
\begin{aligned}
& |e\rangle=\frac{1}{\sqrt{2}}(|1\rangle+|2\rangle), \\
& |o\rangle=\frac{1}{\sqrt{2}}(|1\rangle-|2\rangle),
\end{aligned}
$$

where $|e\rangle$ and $|o\rangle$ are even and odd with respect to the slab center. Because of symmetry, the self-energy and Green's function matrices are diagonal with respect to these MO basis functions. Since the Hamiltonian matrix elements are given by

$$
\begin{aligned}
H_{e e}\left(\mathbf{k}, i \omega_{n}\right)= & -t-2 t\left[\cos \left(k_{x} a\right)+2 \cos \left(k_{y} a\right)\right]+\Re \Sigma_{12}\left(i \omega_{n}\right) \\
& +\Im \Sigma_{11}\left(i \omega_{n}\right), \\
H_{o o}\left(\mathbf{k}, i \omega_{n}\right)= & +t-2 t\left[\cos \left(k_{x} a\right)+2 \cos \left(k_{y} a\right)\right]-\mathfrak{R} \Sigma_{12}\left(i \omega_{n}\right) \\
& +\Im \Sigma_{11}\left(i \omega_{n}\right),
\end{aligned}
$$

the splitting of the $|e\rangle$ and $|o\rangle$ bands due to the abrupt change in $\mathfrak{R} \Sigma_{12}$ at the critical $U$, together with band narrowing 


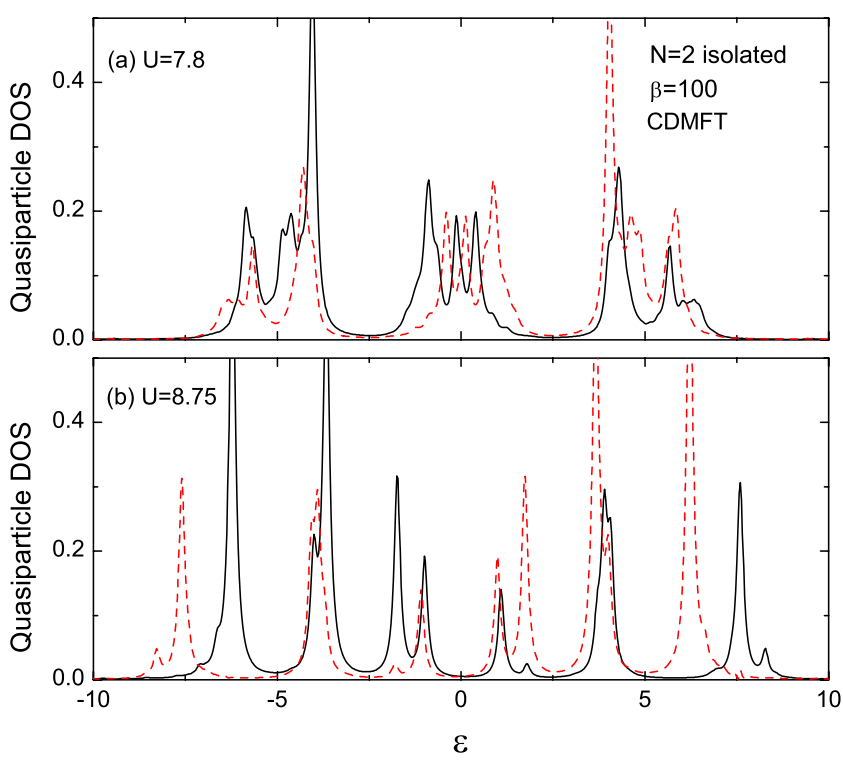

FIG. 5. (Color online) Quasiparticle components of DOS of ED cluster for an isolated two-layer film at $\beta=100$ within CDMFT. Solid curve: $|e\rangle$; dashed curve: $|o\rangle$. (a) $U=7.8$, and (b) $U=8.75$.

caused by $\Im \Sigma_{11}$, gives rise to an insulating gap at $\mu$, between the more than half filled $|e\rangle$ band and the less than half filled $|o\rangle$ band. The solid circles in Fig. 4(b) show the deviation in occupation of the two bands from one half, $n_{e}-1 / 2=1 / 2$ $-n_{o}=\mathfrak{R} G_{12}(\tau=0)$, as a function of $U . n_{e}$ and $n_{o}$ change discontinuously when the system undergoes the MIT, although the magnitude of this jump, $\sim 0.03$, is rather small.

A similar MIT was reported in a recent CDMFT study of Park et al. for the Hubbard model on a 2D square lattice at half-filling. ${ }^{34}$ They employed a $2 \times 2$ cluster to study the effects of short-range correlations on MIT and found that the MIT is accompanied by a splitting of the $\Gamma$ orbital corresponding to $\left(k_{x}, k_{y}\right)=(0,0)$ and the $M$ orbital corresponding to $(\pi, \pi)$ caused by a discontinuous change in the real part of the off-diagonal components of the self-energy connecting nearest-neighbor sites in the cluster.

The opening of the insulating gap can be seen most clearly in the $|e\rangle$ and $|o\rangle$ components of the quasiparticle DOS which are shown in Fig. 5. Since we are concerned here with the MIT, we plot the ED cluster spectra, $-\frac{1}{\pi} \Im G_{j j}^{c l}(\epsilon$ $+i \eta)$ with a broadening parameter $\eta=0.1$, which can be evaluated without requiring extrapolation from Matsubara to real frequencies. In the metallic phase $(U=7.8)$, the spectra of both MO's consist of a quasiparticle peak near $\mu=0$ and upper and lower Hubbard bands. In the insulating phase $(U$ $=8.75$ ), an insulating gap appears near $\mu=0$ which opens via an abrupt change in $\mathfrak{R} \Sigma_{12}$ as discussed above. Although the one-electron Green's function is diagonal with respect to the two MO's, the even and odd bands are coupled via manybody Coulomb interactions. As a result, the quasiparticle DOS of both $|e\rangle$ and $|o\rangle$ bands exhibit considerable spectral weight on both sides of the energy gap.

We point out here that the opening of the gap seen in Fig. 5 is not an artifact caused by the finite size of the bath used in the ED calculation. The near absence of finite-size problems is evident from the perfectly smooth variation of the

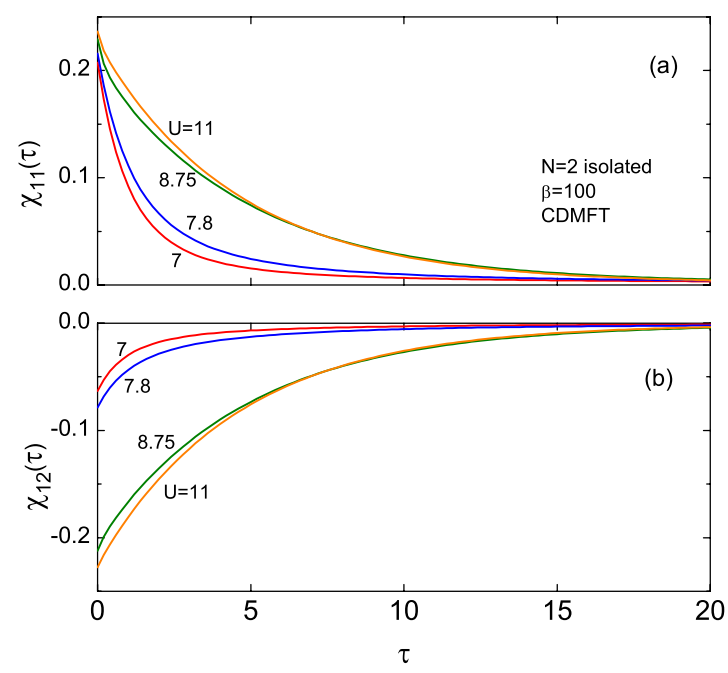

FIG. 6. (Color online) (a) Intralayer and (b) interlayer spin correlations as functions of imaginary time for an isolated two-layer film at $\beta=100$ in the metallic $(U=7,7.8)$ and insulating $(U$ $=8.75,11)$ regimes.

self-energy components even at very small Matsubara frequencies as shown in Fig. 3. This behavior is related to the highly accurate projection of the lattice bath Green's functions $G_{j j^{\prime}}^{0}\left(i \omega_{n}\right)$ onto the corresponding cluster function $G_{j j^{\prime}}^{c l, 0}\left(i \omega_{n}\right)$, as indicated in Eqs. (13) and (14). Evidently, using three bath levels per molecular orbital site, both metallic and insulating features are captured very well. For instance, the linear variation of $\mathfrak{I} \Sigma_{11}\left(i \omega_{n}\right)$ at small $\omega_{n}$ and not too large values of $U$ in Fig. 3 implies the existence of a well-defined quasiparticle peak in the lattice Green's function at $E_{F}$ (not shown here). Thus, the gap opening at larger $U$ is purely related to the rapid increase of $\mathfrak{R} \Sigma_{12}\left(i \omega_{n}\right)$.

To investigate the nature of the MIT in more detail we have calculated the non-local spin correlations characterized by $\chi_{i j}(\tau)=\left\langle\hat{s}_{z i}(\tau) \hat{s}_{z j}(0)+\hat{s}_{z j}(\tau) \hat{s}_{z i}(0)\right\rangle / 2$, where $\tau$ represents imaginary time, and $i$ and $j$ are layer indices. In Fig. 6, we show the intralayer and interlayer components of the spin correlation function in the metallic $(U=7,7.8)$ and insulating $(U=8.75,11)$ regimes. The intralayer component $\chi_{11}$ decays with $\tau$, so that $\chi(\tau=\beta / 2)$ is very small, indicating that the susceptibility, $\chi_{m} \sim \int_{0}^{\beta}\left\langle\hat{s}_{z 1}(\tau) \hat{s}_{z 1}(0)\right\rangle d \tau$, is Pauli-like in both metallic and insulating regions. In the insulating region, $\chi_{11}$ decays less rapidly, implying a larger value of the spin susceptibility. Concerning the interlayer component, as can be seen from Fig. 6, in the metallic range interlayer correlations are much weaker than onsite intralayer correlations. In the insulating region, however, $\chi_{12}(\tau=0) \sim-0.25$, indicating the formation of nonlocal spin singlets between neighboring planes. To see this behavior more clearly, we plot in Fig. 4(c) $\chi_{12}(\tau=0)$ of the isolated two-layer film as a function of $U$ by solid circles. It is seen that this value exhibits a discontinuous change as the system undergoes the MIT.

\section{B. Sandwiched $N=2$ film}

We now consider a two-layer film sandwiched between two semi-infinite noninteracting metallic leads. As stated be- 

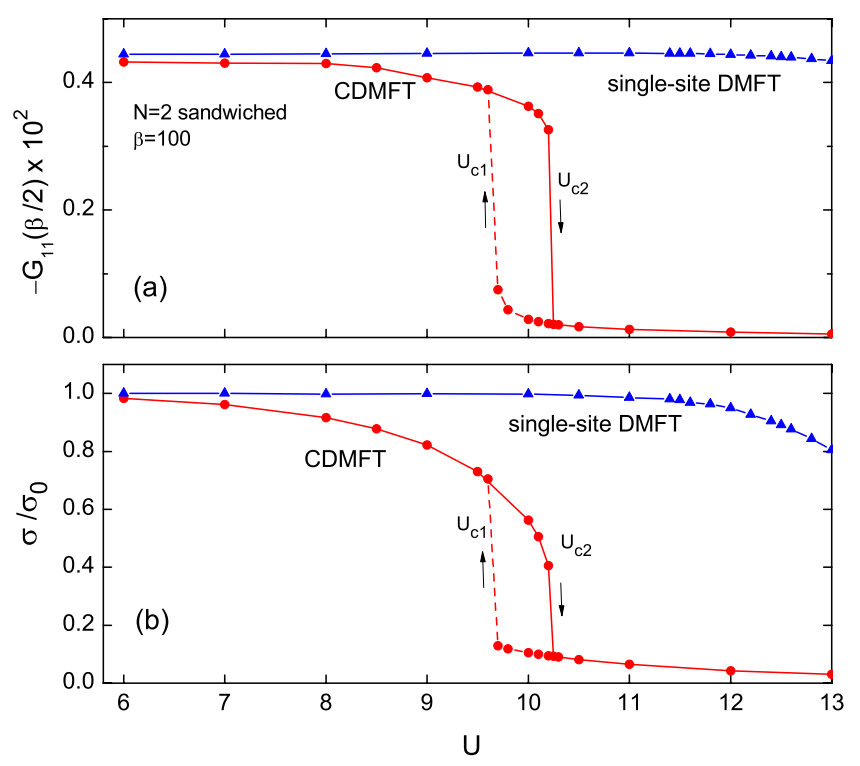

FIG. 7. (Color online) (a) $G_{11}(\beta / 2)$, imaginary-time Green's function at $\beta / 2$, and (b) normalized conductance $\sigma / \sigma_{0}$ in the zerobias limit for a two-layer film sandwiched between two semiinfinite metallic leads as a function of $U$ at $\beta=100$. Solid circles: CDMFT; triangles: single-site DMFT.

fore, the system becomes a half-filled simple cubic crystal with band width $W=12$ when $U=0$. Solid circles and triangles in Fig. 7(a) show $-G_{11}(\beta / 2)$ as a function of $U$ obtained within cluster and single-site DMFT, respectively. In contrast to Fig. 2(a), within single-site DMFT the sandwiched film shows no first-order phase transition and $-G_{11}(\beta / 2)$ remains nearly constant. As recently discussed by Zenia et al. $^{21}$ using the same half-filled single-band Hubbard model in the sandwich geometry combined with single-site DMFT, the coherent peak at $\mu=0$ continues to exist due to the normal-metal proximity effect ${ }^{23}$ if $T \ll T_{F}$. Here, $T_{F}$ is a layer-dependent effective Fermi temperature which decreases exponentially with increasing $U$ and with increasing distance from the lead. They called this phenomenon the appearance of a "fragile" Fermi liquid in a thin Mott-insulator film. ${ }^{21}$ In the present calculation at $\beta=100$ and $N=2$, this condition is clearly fulfilled, explaining why $-G_{11}(\beta / 2)$, the integrated spectral weight around $\mu=0$, remains nearly constant up to $U \sim 11.5$, i.e., at Coulomb energies where the free-standing film would clearly be Mott insulating.

In striking contrast to this single-site DMFT behavior, the CDMFT results for $-G_{11}(\beta / 2)$ shown in Fig. $7($ a) exhibit a hysteresis characteristic of a first-order phase transition. The critical Coulomb energies $U_{c 1}$ and $U_{c 2}$ are shifted by $\sim 1.7$ to larger values as compared with those for the isolated film shown in Fig. 2(a). The nature of this phase transition is essentially the same as that of the isolated film. This is illustrated in Fig. 4 where we plot $\Re \Sigma_{12}\left(i \omega_{0}\right), n_{e}-1 / 2$, and $\chi_{12}(\tau=0)$ as functions of $U$. In close analogy to the isolated two-layer film, the phase transition is accompanied by an abrupt change in $\Re \Sigma_{12}$, which leads to the splitting of the $|e\rangle$ and $|o\rangle$ bands. As in the case of the isolated film, the phase at larger $U$ is characterized by larger negative spin correlations between neighboring planes.

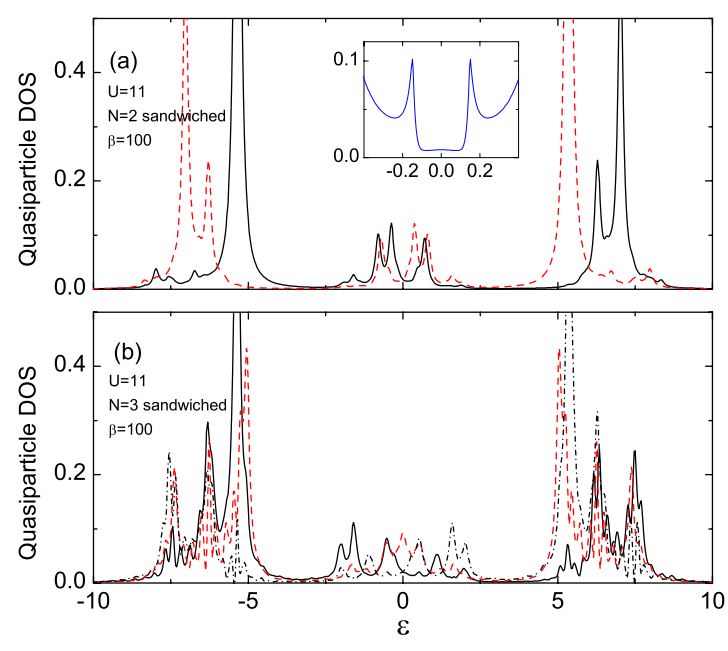

FIG. 8. (Color online) Quasiparticle components of DOS of ED cluster for a sandwiched film at $\beta=100$ within CDMFT. (a) $N=2$. Solid curve: $|e\rangle$; dashed curve: $|o\rangle$. (b) $N=3$. Solid curve: $\left|e_{1}\right\rangle$; dashed curve: $|o\rangle$; dashed-dotted curve: $\left|e_{2}\right\rangle$. The inset in panel (a) shows the total solid DOS for $N=2$ obtained by extrapolating the Green function from Matsubara frequencies to real energies.

In Fig. 8(a), we plot the $|e\rangle$ and $|o\rangle$ components of the ED cluster spectra at $U=11>U_{c 2}$ calculated with the same broadening parameter $\eta$ as in Fig. 5. In contrast to the corresponding DOS within single-site DMFT, which exhibits a quasiparticle peak at $\mu=0$ at the same $U$, the chemical potential in Fig. 8(a) is located between the two DOS peaks resulting from the splitting of the two bands. To see this point more clearly, we evaluate the quasiparticle DOS of the solid by extrapolating the Green's function from Matsubara frequencies to real energies by making use of the extrapolation subroutine ratint. ${ }^{39}$ The inset in Fig. 8(a) shows the obtained spectra near $\mu=0$, which was found to be quite insensitive to the number of Matsubara frequencies used in the extrapolation procedure. It is seen that $\mu$ is located between two sharp DOS peaks at $\epsilon= \pm 0.15$. Characteristic features of this DOS near $\mu$ are very similar to the ones obtained for the 2D Hubbard model in the insulating phase in recent CDMFT studies. ${ }^{32,34}$ As can be seen in the inset, the DOS between the two peaks does not vanish in the present case. Instead, it has a nearly constant positive value, which may be interpreted as arising from the metallic states of both leads penetrating into the central film.

Our CDMFT calculation suggests that, as a result of interplane correlations, the "fragile" Fermi-liquid state in a sandwiched Mott-insulator film as proposed by Zenia et al. ${ }^{21}$ no longer exists, even for a thin film consisting only of two layers. Instead, the system undergoes a phase transition to an energetically more favorable state in which the Fermi-liquid coherence peak at $\mu$ is absent.

Another interesting quantity characterizing the sandwiched film is the conductance of the junction. While the electronic current through a thin film under application of a finite bias voltage between two metallic leads can be evaluated only by performing a nonequilibrium Green's function calculation, ${ }^{40,41}$ the conductance in zero-bias limit can be evaluated from the equilibrium Green's function as ${ }^{42}$ 


$$
\sigma=\frac{d J}{d V}=\frac{2 e^{2}}{h} \int \frac{d \mathbf{k}}{(2 \pi)^{2}} \theta(\mathbf{k}),
$$

where $J$ is the electronic current per unit area, $V$ denotes the bias voltage, and $\theta$ is defined in terms of the lead embedding potentials and the retarded Green's function at $\mu$ :

$$
\theta(\mathbf{k})=4 \operatorname{Tr}\left[\mathfrak{I} \hat{s}^{L}(\mathbf{k}, \mu) \hat{G}(\mathbf{k}, \mu) \mathfrak{I} \hat{s}^{R}(\mathbf{k}, \mu) \hat{G}^{*}(\mathbf{k}, \mu)\right] .
$$

In the non-interacting limit $(U=0), \theta(\mathbf{k})$ coincides with electron transmission rate. In the non-interacting limit, the present system reduces to a three-dimensional (3D) simple cubic lattice without defects. Thus, $\theta(\mathbf{k})$ at $U=0$ is unity or zero depending on whether $\mathbf{k}$ is located inside or outside the projection of the 3D Fermi surface onto the 2D Surface Brillouin zone. In Fig. 7(b) we show the calculated conductance normalized by its value at $U=0, \sigma_{0}$, as a function of $U$. To derive $G_{1, N}(\mathbf{k}, \mu)$ from the Green's function on Matsubara frequencies, we again made use of ratint. ${ }^{39}$ In the single-site DMFT calculation (triangles), the film possesses a perfect conductivity $\left(\sigma / \sigma_{0}=1\right)$ up to $U \sim 11.5$, since the system remains a Fermi liquid due to the normal-state proximity effects as discussed above. Thus, the scattering rate, $\Im \Sigma_{j j}$, vanishes at $\mu=0$. For larger $U, \sigma / \sigma_{0}$ starts to deviate from unity, implying that the condition $T \ll T_{F}$ ceases to hold and the system enters a non-Fermi-liquid state.

The conductance obtained within CDMFT (solid circles) differs qualitatively from the one derived within the singlesite approximation. Whereas the components of the imaginary part of the self-energy vanish at $\mu=0, \mathfrak{R} \Sigma_{12}$ has a finite value at $\mu=0$ as shown in Fig. 3(c). This means that the effective transfer integral between the first and second layers deviates from the bulk value, $t_{j j^{\prime}}^{z}=1$. As a result, electrons incident from the interior of the left or right lead with energy $\mu$ are partly reflected at the interface. As $\mathfrak{R} \Sigma_{12}$ is proportional to $U^{2}$ at small $U, \sigma / \sigma_{0}$ deviates from unity quadratically as a function of U. Furthermore, the conductance drops to much smaller values when the system undergoes a firstorder phase transition at the critical Coulomb energies. The electron conduction in the large- $U$ phase may be understood in terms of electron tunneling through an insulatorlike thin film.

\section{Isolated $N=3$ film}

Next, we investigate a free-standing three-layer film not connected to metallic leads. In the present half-filled threelayer film with particle-hole symmetry, $\mathfrak{R} \Sigma_{j j}=U / 2$ $(j=1 \ldots 3), \mathfrak{I} \Sigma_{12}=\mathfrak{I} \Sigma_{23}=\Re \Sigma_{13}=0$, while the other components, $\mathfrak{I} \Sigma_{11}=\mathfrak{I} \Sigma_{33}, \mathfrak{I} \Sigma_{22}, \mathfrak{R} \Sigma_{12}=\mathfrak{R} \Sigma_{23}, \mathfrak{I} \Sigma_{13}$, are nontrivial functions of Matsubara frequency.

In Figs. 9(a) and 9(b), we plot $-G_{j j}(\beta / 2)$, the imaginarytime Green's function at $\tau=\beta / 2$, for the outer $(j=1,3)$ and middle $(j=2)$ layers, respectively. In single-site DMFT, there exists a single first-order phase transition. All layers become insulating at $U_{c 2}^{s}$ for increasing $U$ and return to a metallic state at $U_{c 1}^{s}$ for decreasing $U$. As in the case of $N=2$, the imaginary part of the local self-energy $\Sigma_{j j}$ is proportional to $\omega_{n}$ at small $\omega_{n}$ in the metallic phase, while it diverges as $1 / \omega_{n}$ in the insulating phase (not shown).
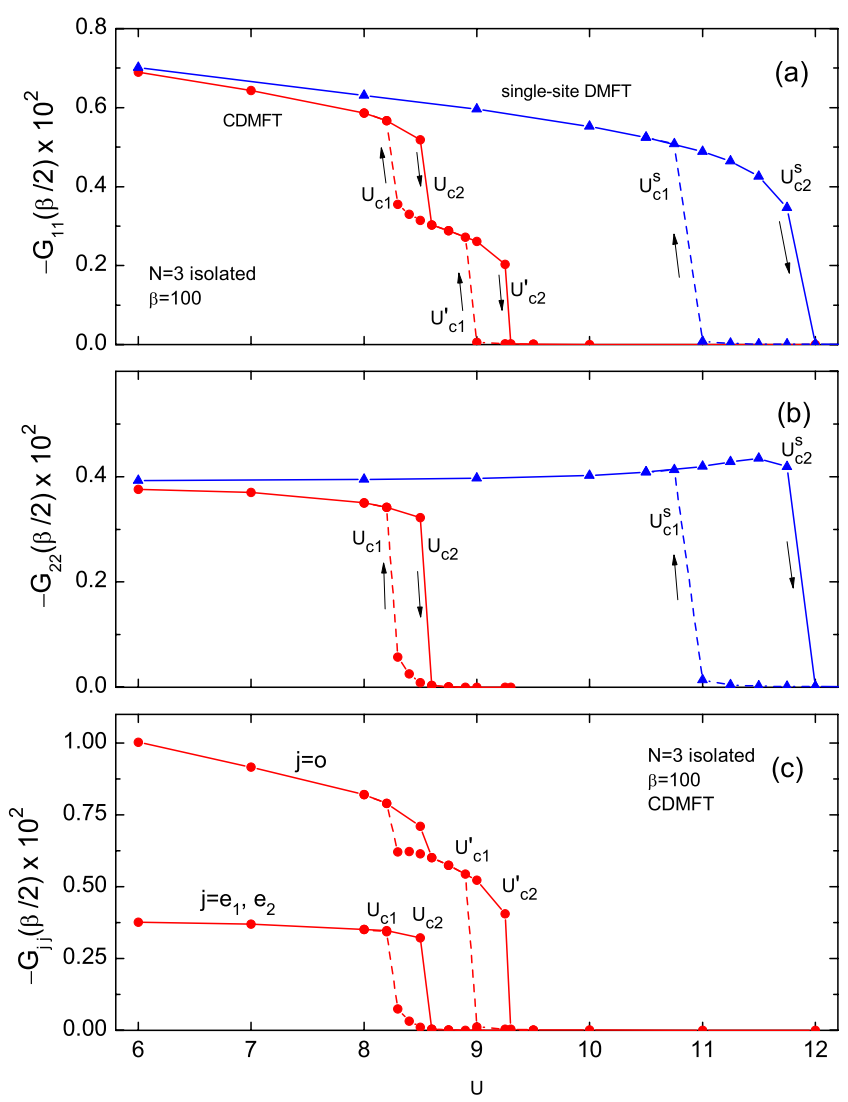

FIG. 9. (Color online) $G_{j j}(\beta / 2)$ of an isolated three-layer film as a function of Coulomb energy $U$ at $\beta=100$ for (a) outer layer $(j=1,3)$, (b) middle layer $(j=2)$, and (c) three molecular orbitals. Solid circles: CDMFT; triangles: single-site DMFT.

In striking contrast, the CDMFT results reveal two successive first-order phase transitions. The MIT of the central layer $(j=2)$ takes place at a smaller critical Coulomb energy, $U_{c 1(c 2)}[$ see panel (b)]. At these Coulomb energies, the integrated spectral weight near $\mu=0$ of the outer layers $(j=1,3)$ is approximately halved [see panel (a)].

The MIT of these layers occurs at larger Coulomb energies, $U_{c 1(c 2)}^{\prime}$. Apart from this qualitative difference, the MIT within CDMFT is completed at much lower $U$ values than those predicted by single-site DMFT, just as in the case of the isolated two-layer film discussed above.

In the present model, the self-energy and Green's function matrices of the three-layer film are block diagonal with respect to three MO basis functions:

$$
\begin{aligned}
& \left|e_{1}\right\rangle=\frac{1}{2}(|1\rangle+\sqrt{2}|2\rangle+|3\rangle), \\
& \left|e_{2}\right\rangle=\frac{1}{2}(|1\rangle-\sqrt{2}|2\rangle+|3\rangle),
\end{aligned}
$$

are even with respect to the slab center and form a $2 \times 2$ block, while 

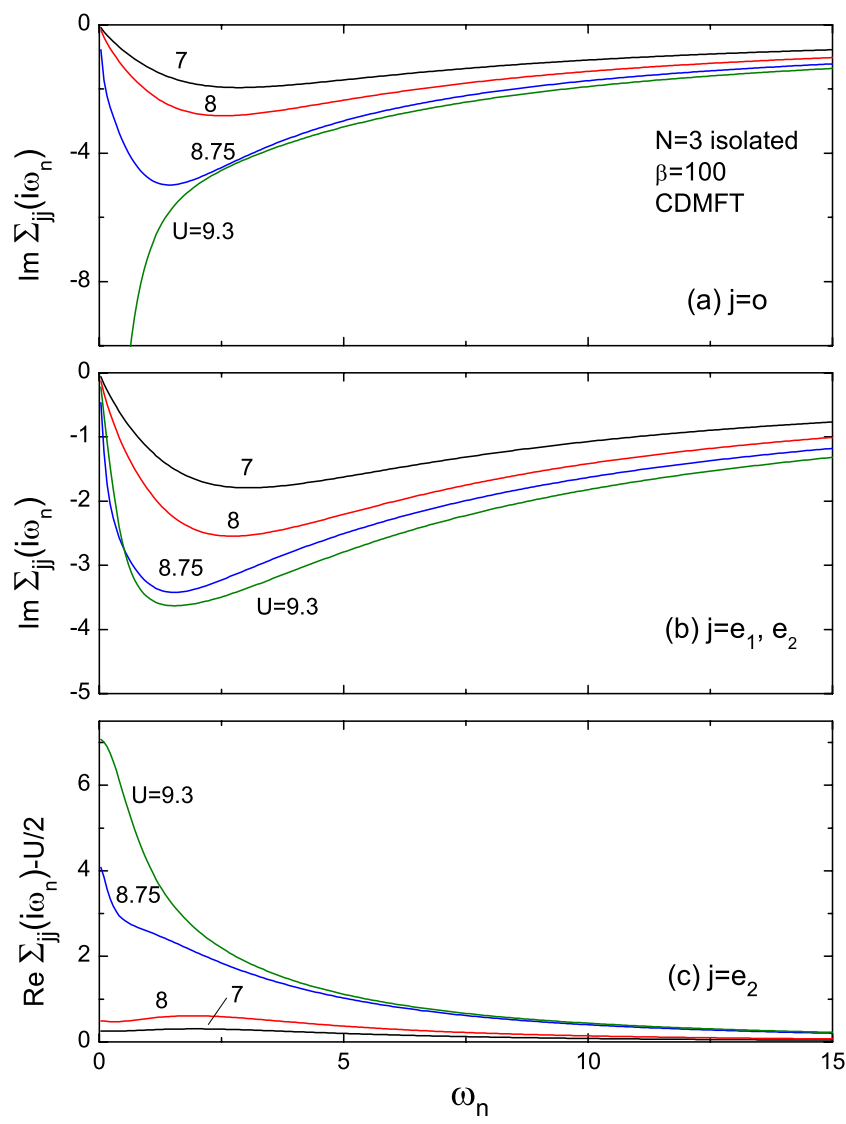

FIG. 10. (Color online) Self-energy components of an isolated 3-layer film obtained by CDMFT as a function of Matsubara frequency $\omega_{n}$ at $\beta=100$ in molecular orbital basis. (a) $\mathfrak{I} \Sigma_{o o}$, (b) $\mathfrak{I} \Sigma_{e_{1} e_{1}}$, and (c) $\Re \Sigma_{e_{2} e_{2}}-U / 2$.

$$
|o\rangle=\frac{1}{\sqrt{2}}(|1\rangle-|3\rangle),
$$

is odd with respect to the slab center and forms a $1 \times 1$ block. $\left|e_{1}\right\rangle$ and $\left|e_{2}\right\rangle$ constitute eigenstates of the noninteracting three-layer film, and in this limit the $2 \times 2$ matrix of the Green's function of the even orbitals becomes diagonal. For finite $U$, the Green's function is not diagonal because of the nonvanishing off-diagonal Hamiltonian element, giving rise to a finite $-\Sigma_{e_{1} e_{2}}$.

In Fig. 9(c), we replot $-G_{j j}(\beta / 2)$ of the isolated threelayer film using the MO basis. Evidently, the phase-transition between $U_{c 1}$ and $U_{c 2}$ can be regarded as the MIT of the even orbitals, while the second one between $U_{c 1}^{\prime}$ and $U_{c 2}^{\prime}$ is the transition of the odd orbital $|o\rangle$. As noted above, the even and odd bands are coupled via many-body Coulomb interactions. As a consequence, $-G_{o o}(\beta / 2)$, the integrated spectral weight of the $|o\rangle$ band around $\mu=0$, exhibits a discontinuous change also at $U_{c 1}$ and $U_{c 2}$.

Interestingly, the nature of these two successive phase transitions differs qualitatively. In Fig. 10(a), we show the imaginary part of the self-energy for the $|o\rangle$ band, $\Sigma_{o o}=\Sigma_{11}$ $-\Sigma_{13}$, as a function of Matsubara frequency for four $U$ values. Since the quasiparticle DOS of this band is symmetric with respect to $\mu=0$, the mechanism with which it undergoes a MIT is similar to that of a single-layer film, or that of a multilayer film within the single-site DMFT treatment. Namely, $\Im \Sigma_{o o}$ is proportional to $\omega_{n}$ at small $\omega_{n}$ up to $U_{c 2(c 1)}^{\prime}$ for increasing (decreasing) $U$, while it diverges as $1 / \omega_{n}$ for larger $U$ values $(U=9.3)$, thus opening an energy gap. $\Im \Sigma_{11}$ and $\mathfrak{I} \Sigma_{13}$, whose difference equals $\mathfrak{I} \Sigma_{o o}$, exhibit the same behavior as a function of $\omega_{n}$ : they fall to zero or diverge as $1 / \omega_{n}$ in the limit of $\omega_{n} \rightarrow 0$, depending on whether $U$ is located below or above the critical Coulomb energies, respectively. In the insulating phase, it has been found that they behave at small $\omega_{n}$ as

$$
\begin{gathered}
\Im \Sigma_{11}=\frac{A}{\omega_{n}}+\alpha_{11} \omega_{n}, \\
\Im \Sigma_{13}=\frac{-A}{\omega_{n}}+\alpha_{13} \omega_{n} .
\end{gathered}
$$

In contrast, the transition of the two even bands is caused essentially by the splitting of the $\left|e_{1}\right\rangle$ and $\left|e_{2}\right\rangle$ bands. As shown in Fig. 10(b), the diagonal components of the imaginary part of the self energy,

$$
\mathfrak{I} \Sigma_{e_{1} e_{1}}=\mathfrak{I} \Sigma_{e_{2} e_{2}}=\frac{1}{2}\left(\mathfrak{I} \Sigma_{11}+\mathfrak{I} \Sigma_{22}+\mathfrak{I} \Sigma_{13}\right)
$$

do not diverge as a function of $\omega_{n}$ even in the insulating phase and tend to zero in the limit of $\omega_{n} \rightarrow 0$. Here, as seen from Eq. (28), the diverging terms of $\Sigma_{11}$ and $\Sigma_{13}$ cancel each other in Eq. (29), which explains why $I \Sigma_{e_{1} e_{1}}$ tends to zero even for $U=9.3>U_{c 2}^{\prime}$.

On the other hand, their real components,

$$
\begin{aligned}
& \Re \Sigma_{e_{1} e_{1}}=\frac{U}{2}+\sqrt{2} \mathfrak{R} \Sigma_{12}, \\
& \Re \Sigma_{e_{2} e_{2}}=\frac{U}{2}-\sqrt{2} \mathfrak{R} \Sigma_{12},
\end{aligned}
$$

exhibit a discontinuous change at the critical Coulomb energies. To illustrate this behavior, we plot in Fig. 10(c) $\Re \Sigma_{e_{2} e_{2}}-U / 2$ for four $U$ values. Its value at small $\omega_{n}$ for $U$ $=8.75$ and 9.3 in the insulating phase is seen to be much larger than at $U=7$ and $U=8$ in the metallic phase. This is shown more clearly in Fig. 11(a), where we plot $\Re \Sigma_{12}$ of the isolated three-layer film at the first Matsubara frequency $\omega_{0}$. $\mathfrak{R} \Sigma_{12}$, which determines the splitting between $\mathfrak{R} \Sigma_{e_{1} e_{1}}$ and $\Re \Sigma_{e_{2} e_{2}}$ as indicated by Eq. (30), is seen to exhibit a discontinuous change at $U_{c 1(c 2)}$, giving rise to a downward (upward) shift of the quasiparticle DOS of $\left|e_{1}\right\rangle\left(\left|e_{2}\right\rangle\right)$ and the concomitant opening of an insulating gap. This mechanism is similar to that of the isolated two-layer film discussed in the preceding subsection. In the present three-layer film, the Green's function is not diagonal with respect to the even orbitals. However, since the off-diagonal Hamiltonian matrix element, 

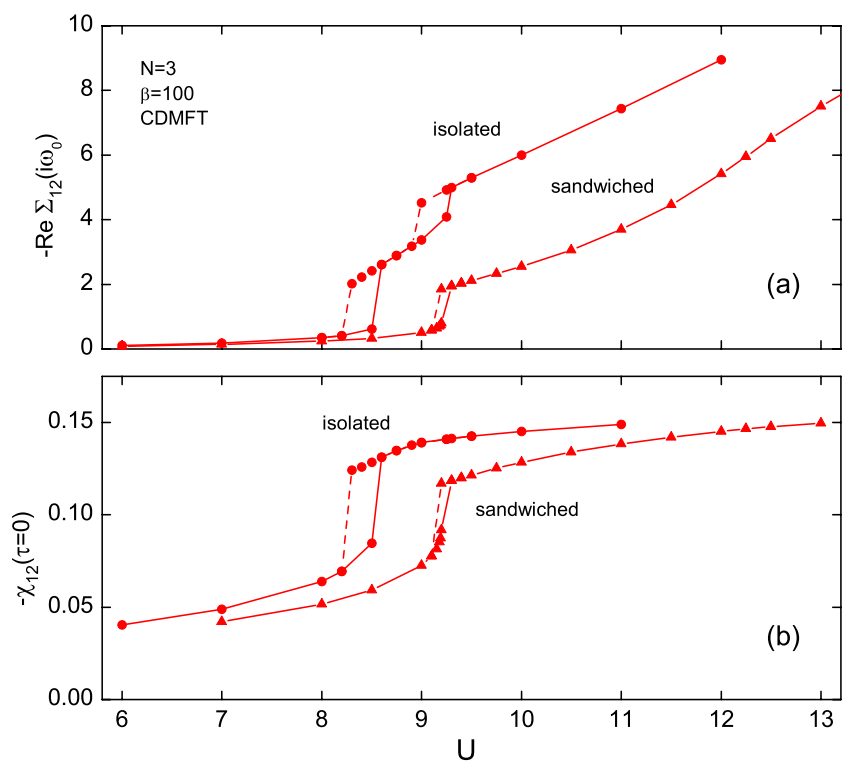

FIG. 11. (Color online) (a) $\mathfrak{R} \Sigma_{12}$ at the first Matsubara frequency $\omega_{0}=\pi / \beta$ and (b) $\chi_{12}(\tau=0)$, interlayer spin correlation at $\tau$ $=0$, as a function of $U$ at $\beta=100$ by CDMFT. Solid circles and triangles correspond to the isolated and sandwiched three-layer films, respectively.

$$
\Sigma_{e_{1} e_{2}}=\frac{i}{2}\left(\Im \Sigma_{11}-\Im \Sigma_{22}+\Im \Sigma_{13}\right),
$$

approaches zero in the limit of $\omega_{n} \rightarrow 0$ in the $U$ range relevant to the MIT of the even bands, the Green's function and consequently the quasiparticle DOS around $\mu=0$ are determined essentially by the diagonal elements of the Hamiltonian matrix.

In Fig. 11(b), we plot the interlayer spin correlation function at $\tau=0, \chi_{12}(0)$, as a function of $U$. As is seen, the MIT of the even orbitals between $U_{c 1}$ and $U_{c 2}$ is accompanied by a discontinuous change of $\chi_{12}(\tau=0)$. The phase on the larger $U$ side is characterized by enhanced negative spin correlations between neighboring layers as in the case of the isolated two-layer film, although its absolute value $\left|\chi_{12}(0)\right| \sim 0.15$ is considerably smaller than the corresponding one for $N=2$ shown in Fig. 4(c). On the other hand, $\chi_{12}(0)$ does not exhibit a discontinuous jump at the second phase transition between $U_{c 1}^{\prime}$ and $U_{c 2}^{\prime}$, although its slope as a function of $U$ changes slightly for $U>U_{c 2}^{\prime}$. Since this transition can be regarded as a Mott transition of a 2D single Hubbard band originating from the odd orbital $|o\rangle$, it does not necessarily involve abrupt changes in nonlocal spin correlations in the surface normal direction.

\section{Sandwiched $N=3$ film}

Finally, we consider a three-layer film sandwiched between two semi-infinite non-interacting metallic leads. In Figs. 12(a) and 12(b), we show $-G_{j j}(\beta / 2)$ as a function of $U$ for the outer $(j=1,3)$ and central $(j=2)$ layers, respectively. Within single-site DMFT, there is no first-order phase transition, just as in the case $N=2$. For the outer two layers, $-G_{j j}(\beta / 2)$, remains nearly constant up to $U \sim 12$ because of
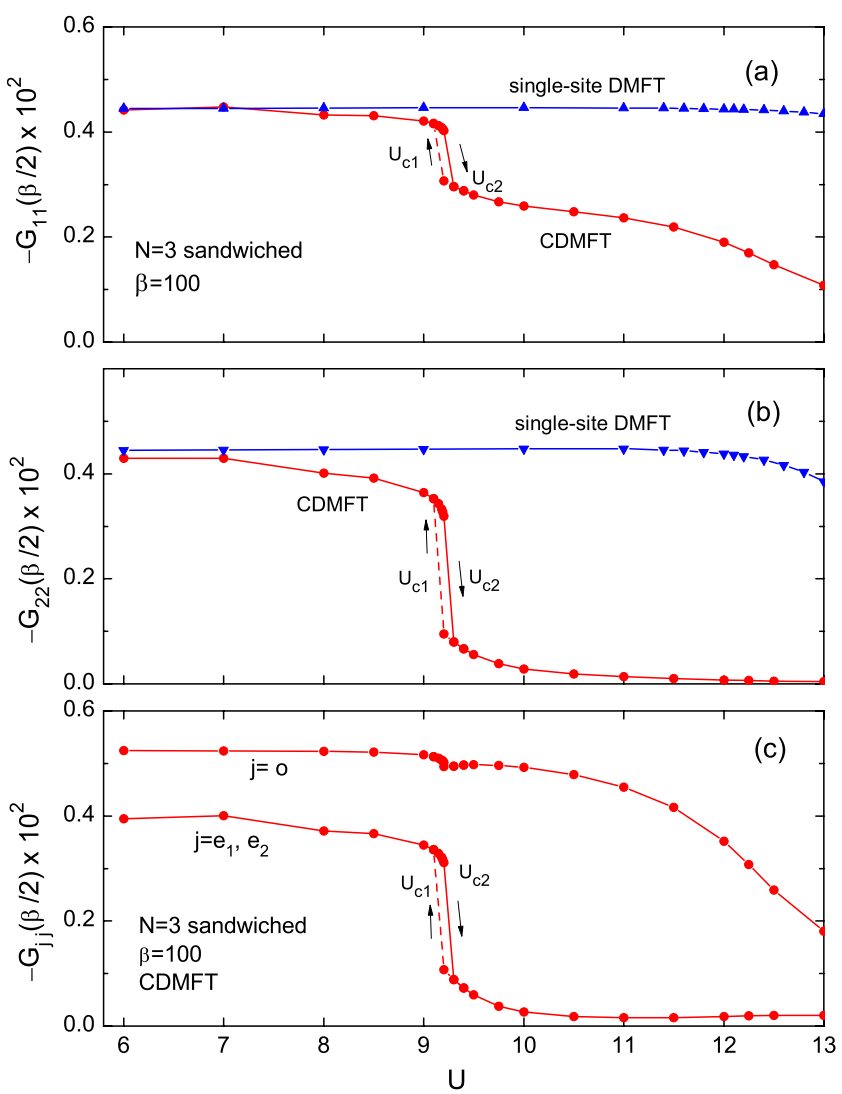

FIG. 12. (Color online) $G_{j j}(\beta / 2)$ of a sandwiched three-layer film as a function of Coulomb energy $U$ at $\beta=100$ for (a) outer layer $(j=1,3)$, (b) middle layer $(j=2)$, and (c) three molecular orbitals. Solid circles: CDMFT; triangles: single-site DMFT.

the normal-metal proximity effects. For the central layer, $-G_{j j}(\beta / 2)$ deviates slightly at around $U=11$ from a constant, indicating that the condition $T \ll T_{F}$ ceases to hold near this $U$ value, so that this layer enters a non-Fermi-liquid state. This is not unexpected, since the layer-dependent Fermi temperature $T_{F}$ for the central layer is smaller than that of the layers located next to the metallic leads.

Within CDMFT, on the other hand, a first-order phase transition does exist. Nevertheless, it is a single transition at $U_{c 1(c 2)}$, in contrast to the successive transitions found for the isolated three-layer film, as shown in Fig. 9. While the quasiparticle DOS of the central layer drops strongly at these critical Coulomb energies, in the outer layers this reduction is much less pronounced. At larger $U,-G_{j j}(\beta / 2)$ of the outer layers decreases gradually without undergoing a second phase transition. These behaviors can be understood by projecting the Green's function on the three MO's. In Fig. 12(c), we plot $-G_{j j}(\beta / 2)$ for the orbitals defined in Eqs. (26) and (27). Evidently, the first-order phase transition originates mainly from the two even bands, whose integrated spectral weight near $\mu$ drops strongly at $U_{c 1(c 2)}$. It does not vanish completely at larger $U$ values, since the metallic states of the leads weakly penetrate into the sandwiched film. As the even and odd bands couple with each other via many-body Coulomb interactions, $-G_{o o}(\beta / 2)$ also exhibits a small discontinuous change at $U_{c 1(c 2)}$. However, at larger $U$ values, $-G_{o o}(\beta / 2)$ decreases only slightly, without any phase transi- 


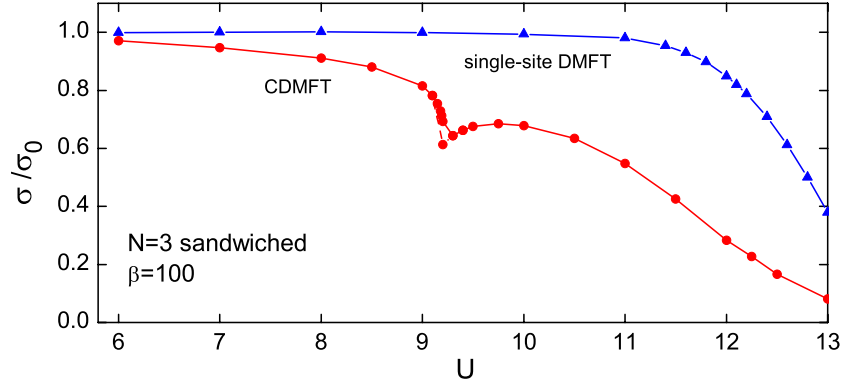

FIG. 13. (Color online) Normalized conductance in the zerobias limit for a three-layer film sandwiched between two semiinfinite metallic leads as a function of $U$ at $\beta=100$.

tion, in contrast to the isolated three-layer film.

The nature of the phase transition of the even bands is essentially the same as that of the isolated film. As shown in Fig. 11 by triangles, the real part of the off-diagonal components of the self energy, $\Sigma_{12}=\Sigma_{23}$, exhibits a discontinuous change at the critical Coulomb energy, $U_{c 1(c 2)}$, which leads to the splitting of the more than half-filled $\left|e_{1}\right\rangle$ band and the less than half-filled $\left|e_{2}\right\rangle$ band, and simultaneously to an abrupt decrease in the quasiparticle DOS at $\mu=0$ of both bands. At the same time, the interlayer spin correlation function at $\tau$ $=0, \chi_{12}(0)$, exhibits a discontinuous change similarly to the isolated three-layer film. On the other hand, as the quasiparticle DOS of the $|o\rangle$ band is symmetric with respect to $\mu$ $=0$ and its quasiparticle peak is fixed to $\mu$, the $|o\rangle$ band behaves similarly to a single-layer film or a multilayer film within the single-site DMFT treatment. That is, it is subject to the normal-metal proximity effects and its quasiparticle peak at $\mu=0$ persists to exist as long as $T \ll T_{F}$. This condition seems to be satisfied in the present case, which explains why $-G_{o o}(\beta / 2)$ remains nearly constant up to $U \sim 10$. To verify these points, we plot in Fig. 8(b) the three MO components of the ED cluster spectra at $U=11>U_{c 2}$. As is seen, the DOS of the even bands exhibits a minimum at $\mu=0$ due to the splitting of the two even bands, whereas the $|o\rangle$ component of DOS has a peak at $\mu=0$.

In Fig. 13, we show the calculated conductance of the half-filled three-layer film in the zero-bias limit as a function of $U$, normalized by its value at $U=0, \sigma_{0}$. Within single-site DMFT, $\sigma / \sigma_{0}$ is unity up to around $U=11$, since $\Im \Sigma_{j j}$ at $\mu$ vanishes as long as the system is in a Fermi-liquid state. $\sigma / \sigma_{0}$ starts to deviate from unity at around $U=11$ as the central layer deviates from Fermi-liquid behavior. Beyond $U \sim 12, \sigma$ decreases rather quickly, probably because both the outer layers also enter a non-Fermi-liquid state. The behavior of the conductance obtained within CDMFT is qualitatively different from the one found in single-site DMFT. As in the case $N=2, \mathfrak{R} \Sigma_{12}\left(\Re \Sigma_{23}\right)$ at $\mu=0$, which increases quadratically with $U$, contributes to the scattering of conduction electrons and causes a quadratic deviation of $\sigma / \sigma_{0}$ from unity as a function of $U$. In addition, the first-order phase transition of the even bands at the critical Coulomb energies gives rise to a discontinuous change in $\sigma / \sigma_{0}$

\section{E. Discussion}

So far we presented results for $N=2$ and 3 . To extend the CDMFT calculation to a four-layer film with 3 bath orbitals per layer, one has to treat 16 orbitals in the ED formalism, which is beyond our numerical capacity. We can complete the calculation for $N=4$ by reducing the number of bath orbitals per layer to 2. But even in this case, calculating a five-layer film requires matrix diagonalization for a 15orbital ED cluster, which is very demanding. Instead, we give a brief discussion on the MIT of the thicker film at half-filling. For $N=2$ and 3, it has been demonstrated that it is useful to introduce the MO basis set to understand the nature of MIT. For the isolated $N$-layer film, the $l$ th $\mathrm{MO}$, $\left|m_{l}\right\rangle,(1 \leq l \leq N)$ may be defined by

$$
\left|m_{l}\right\rangle=\frac{1}{A_{l}} \sum_{j=1}^{N} \sin \left(\frac{\pi l}{N+1} j\right)|j\rangle,
$$

where $A_{l}$ denotes the normalization factor and $|j\rangle$ on the right-hand side is the basis function of layer $j$. Considering the energy dispersion in the plane, when $U=0$, each $\mathrm{MO}$ forms a $2 \mathrm{D}$ energy band with band width $8 t$ centered at

$$
E_{m_{l}}=-2 t \cos \left(\frac{\pi l}{N+1}\right) \text {. }
$$

These energy bands are classified into even and odd ones with respect to the reflection symmetry about the center plane of the film. The Hamiltonian, Green's function, and self-energy matrices are block diagonal with respect to the even and odd bands.

The first question is whether MIT's of both modes take place at the same critical Coulomb energy $U$ as in the case of $N=2$, or selectively at two $U$ values, as in the case of $N=3$. If $N=2 M$, the $N$ bands are classified into $M$ band pairs. Each pair is made of one even band and one odd band, where the DOS of the odd band is obtained by reflecting that of the even band with respect to $\mu=0$. Namely, if we denote the quasiparticle DOS of the even bands by $\rho_{e}(\epsilon)$ and that of the odd bands by $\rho_{o}(\epsilon)$, they satisfy $\rho_{e}(\epsilon)=\rho_{o}(-\epsilon)$. At half filling, this property should hold irrespective of $U$. Accordingly, the opening of the insulating gap in $\rho_{e}(\epsilon)$ at $\mu=0$ necessarily implies the gap opening in $\rho_{o}(\epsilon)$. Thus, the even and odd modes must undergo a single MIT at the same $U$. On the other hand, if $N=2 M+1$, the $N$ bands are classified into the $M+1$ even and $M$ odd bands. The symmetry implies that $\rho_{e}(\epsilon)=\rho_{e}(-\epsilon)$ and $\rho_{o}(\epsilon)=\rho_{o}(-\epsilon)$, but there is no particular relationship between the two functions. Thus, it might happen that the MIT's of the even and odd modes occur at different $U$ values as in the case of $N=3$.

The second question concerns the mechanism of the MIT. For $N=2$ and 3, we found that there are two gap-opening mechanisms: Either the imaginary part of the local selfenergy diverges, or the real part of the off-diagonal selfenergy components between nearest-neighbor layers exhibits a discontinuous change in the limit of $\omega_{n} \rightarrow 0$. If $N=2 M+1$ and $M$ is an odd integer, among the $M$ odd bands, the $(M$ $+1) / 2$ th odd band is centered at $\mu=0$. Since the second mechanism works to split a pair of bands on both sides of $\mu=0$ toward higher and lower energies, it will not be effective to the $(M+1) / 2$ th band, which has no partner band and whose DOS is centered at $\mu=0$. Thus, we expect that all the odd bands undergo a MIT via the divergence of the local 
self-energy. In the same way, if $N=2 M+1$ and $M$ is an even integer, the DOS of the $(M+2) / 2$ th even band is centered at $\mu=0$, and it is expected that all the even bands undergo a MIT via the divergence of the local self-energy. For the other cases, namely, the even and odd bands when $N=2 M$, or the even bands when $N=2 M+1$ ( $M$ odd), or the odd bands when $N=2 M+1$ ( $M$ even), it is not excluded that the splitting of the pairs of bands by the second mechanism gives rise to an insulating gap at $\mu=0$. However, in these case, the separation of the couple of bands, which are on both sides of $\mu=0$ and the closest to $\mu=0$, becomes smaller with increasing $N$, which would make the gap opening through the splitting of the two bands more difficult. Thus, we presume that MIT by the second mechanism may be realized only at small $N$ where the separation between neighboring bands is relatively large. Returning to the first question, if the second mechanism does not take place and the insulating gap is opened by only one mechanism, i.e., by the divergence of local selfenergies, the even and odd bands may undergo a single MIT not only for $N=2 M$ but also for $N=2 M+1$.

\section{SUMMARY}

We investigated the effect of interplanar Coulomb correlations on the electronic structure of strongly correlated heterostructures by applying the cluster DMFT method combined with finite-temperature exact diagonalization. As a model system, we considered a half-filled single-band Hubbard model in a thin film geometry, which is either free standing or sandwiched between noninteracting semi-infinite metallic leads. The 1D atomic chain oriented normal to the layer plane is chosen as a cluster unit in solving the quantum impurity problem. Thus, the off-diagonal components of the correlation-induced self-energy between layers are fully taken into consideration. Intraplanar intersite correlations are neglected so far for computational reasons.

We have shown that, as a result of interlayer spatial fluctuations, the nature of the metal-insulator transition in these correlated heterostructures is significantly more complex than in the usual single-site DMFT description. In particular, we have demonstrated that, in isolated thin films, two types of first-order metal-insulator phase transitions can occur. One is a conventional one in which the imaginary part of the local self-energy of strongly correlated atomic sites diverges. The other is absent in previous single-site DMFT studies of heterostructures and is associated with a discontinuous change of the real part of the off-diagonal self-energy components between neighboring layers. This mechanism gives rise to a splitting of the even and odd molecular orbital bands $(N$ $=2$ ), or a splitting of the two even molecular orbital bands $(N=3)$ of the correlated thin film and eventually to the opening of an insulating gap. Depending on the number of layers constituting the film, either one or both of these two types of phase transitions may take place.

For thin films sandwiched between metallic leads, the conventional type of phase transition disappears because of the normal-metal proximity effects. Nevertheless, this proximity effect is much weaker than in single-site DMFT. The second type of phase transition continues to exist and influences significantly the electronic properties of the film. For example, the normal component of the conductance may exhibit a jump at critical Coulomb energies. The key point of these results is that, because of interlayer Coulomb fluctuations in the strongly correlated film, the fragile Fermi liquid that is the hallmark of single-site DMFT treatments, is either destroyed or greatly weakened. Thus, the metallicity induced in the Mott insulator via the neighboring leads strongly reduced. It would be interesting to compare these results due to interplanar correlations with analogous effects caused by spatial fluctuations within the atomic planes of the correlated film.

\section{ACKNOWLEDGMENTS}

The work of H.I. was supported by the Grand-in-Aid for Scientific Research (Grant No. 20540191) from the Japan Society for Promotion of Science.
${ }^{1}$ A. Ohtomo and H. Y. Hwang, Nature (London) 427, 423 (2004).

${ }^{2}$ C. H. Ahn, K. M. Rabe, and J.-M. Triscone, Science 303, 488 (2004)

${ }^{3}$ H. Yamada, Y. Ogawa, Y. Ishii, H. Sato, M. Kawasaki, H. Akoh, and Y. Tokura, Science 305, 646 (2004).

${ }^{4}$ E. Dagotto, Science 318, 1076 (2007).

${ }^{5}$ N. Reyren, S. Thiel, A. D. Caviglia, L. Fitting Kourkoutis, G. Hammerl, C. Richter, C. W. Schneider, T. Kopp, A.-S. Rüetschi, D. Jaccard, M. Gabay, D. A. Muller, J.-M. Triscone, and J. Mannhart, Science 317, 1196 (2007).

${ }^{6}$ A. Ohtomo, D. A. Muller, J. L. Grazul, and H. Y. Hwang, Nature (London) 419, 378 (2002).

${ }^{7}$ M. Takizawa, H. Wadati, K. Tanaka, M. Hashimoto, T. Yoshida, A. Fujimori, A. Chikamatsu, H. Kumigashira, M. Oshima, K. Shibuya, T. Mihara, T. Ohnishi, M. Lippmaa, M. Kawasaki, H. Koinuma, S. Okamoto, and A. J. Millis, Phys. Rev. Lett. 97,
057601 (2006).

${ }^{8}$ L. Fitting Kourkoutis, Y. Hotta, T. Susaki, H. Y. Hwang, and D. A. Muller, Phys. Rev. Lett. 97, 256803 (2006).

${ }^{9}$ H. Wadati, Y. Hotta, A. Fujimori, T. Susaki, H. Y. Hwang, Y. Takata, K. Horiba, M. Matsunami, S. Shin, M. Yabashi, K. Tamasaku, Y. Nishino, and T. Ishikawa, Phys. Rev. B 77, 045122 (2008).

${ }^{10}$ T. Higuchi, Y. Hotta, T. Susaki, A. Fujimori, and H. Y. Hwang, Phys. Rev. B 79, 075415 (2009).

${ }^{11}$ M. Potthoff and W. Nolting, Phys. Rev. B 59, 2549 (1999); 60, 7834 (1999).

${ }^{12}$ See also M. Potthoff, Phys. Rev. B 64, 165114 (2001); S. Schwieger, M. Potthoff, and W. Nolting, ibid. 67, 165408 (2003).

${ }^{13}$ A. Georges, G. Kotliar, W. Krauth, and M. J. Rozenberg, Rev. Mod. Phys. 68, 13 (1996). 
${ }^{14}$ For recent reviews, see K. Held, Adv. Phys. 56, 829 (2007); G. Kotliar, S. Y. Savrasov, K. Haule, V. S. Oudovenko, O. Parcollet, and C. A. Marianetti, Rev. Mod. Phys. 78, 865 (2006).

${ }^{15}$ S. Okamoto and A. J. Millis, Nature (London) 428, 630 (2004).

${ }^{16} \mathrm{~S}$. Okamoto and A. J. Millis, Phys. Rev. B 70, 241104(R) (2004).

${ }^{17}$ S. Okamoto and A. J. Millis, Phys. Rev. B 72, 235108 (2005).

${ }^{18}$ J. K. Freericks, Phys. Rev. B 70, 195342 (2004).

${ }^{19}$ J. K. Freericks, V. Zlatić, and A. M. Shvaika, Phys. Rev. B 75, 035133 (2007).

${ }^{20}$ L. Chen and J. K. Freericks, Phys. Rev. B 75, 125114 (2007).

${ }^{21}$ H. Zenia, J. K. Freericks, H. R. Krishnamurthy, and Th. Pruschke, Phys. Rev. Lett. 103, 116402 (2009).

${ }^{22}$ W.-Ch. Lee and A. H. MacDonald, Phys. Rev. B 74, 075106 (2006); 76, 075339 (2007).

${ }^{23}$ R. W. Helmes, T. A. Costi, and A. Rosch, Phys. Rev. Lett. 101, 066802 (2008).

${ }^{24}$ S. Yunoki, A. Moreo, E. Dagotto, S. Okamoto, S. S. Kancharla, and A. Fujimori, Phys. Rev. B 76, 064532 (2007).

${ }^{25}$ W. Heindl, Th. Pruschke, and J. Keller, J. Phys.: Condens. Matter 12, 2245 (2000).

${ }^{26}$ I. González, S. Okamoto, S. Yunoki, A. Moreo, and E. Dagotto, J. Phys.: Condens. Matter 20, 264002 (2008).

${ }^{27}$ T. Oka and N. Nagaosa, Phys. Rev. Lett. 95, 266403 (2005).

${ }^{28}$ S. S. Kancharla and E. Dagotto, Phys. Rev. B 74, 195427
(2006).

${ }^{29}$ A. Rüegg, S. Pilgram, and M. Sigrist, Phys. Rev. B 75, 195117 (2007).

${ }^{30}$ H. Ishida and A. Liebsch, Phys. Rev. B 79, 045130 (2009).

${ }^{31}$ G. Kotliar, S. Y. Savrasov, G. Pálsson, and G. Biroli, Phys. Rev. Lett. 87, 186401 (2001).

${ }^{32}$ B. Kyung, S. S. Kancharla, D. Sénéchal, A.-M. S. Tremblay, M. Civelli, and G. Kotliar, Phys. Rev. B 73, 165114 (2006).

${ }^{33}$ Y. Z. Zhang and M. Imada, Phys. Rev. B 76, 045108 (2007).

${ }^{34}$ H. Park, K. Haule, and G. Kotliar, Phys. Rev. Lett. 101, 186403 (2008).

${ }^{35}$ J. E. Inglesfield, Comput. Phys. Commun. 137, 89 (2001).

${ }^{36}$ D. Kalkstein and P. Soven, Surf. Sci. 26, 85 (1971).

${ }^{37}$ M. Caffarel and W. Krauth, Phys. Rev. Lett. 72, 1545 (1994).

${ }^{38}$ C. A. Perroni, H. Ishida, and A. Liebsch, Phys. Rev. B 75, 045125 (2007).

${ }^{39}$ W. H. Press, S. A. Teukolsky, W. T. Vetterling, and B. P. Flannery, Numerical Recipes in Fortran 77 (Cambridge University Press, New York, 1986-1992), p. 106; J. Stoer and R. Burlisch, Introduction to Numerical Analysis (Springer, New York, 1980).

${ }^{40}$ S. Okamoto, Phys. Rev. Lett. 101, 116807 (2008).

${ }^{41} \mathrm{~S}$. Datta, Electronic Transport in Mesoscopic Systems (Cambridge University Press, Cambridge, England, 1995).

${ }^{42}$ Y. Meir and N. S. Wingreen, Phys. Rev. Lett. 68, 2512 (1992). 